\title{
Generalized Weyl's theorem and quasi-affinity
}

\author{
by \\ Pietro Aiena (Palermo) and Mohammed Berkani (Oujda)
}

\begin{abstract}
A bounded operator $T \in L(X)$ acting on a Banach space $X$ is said to satisfy generalized Weyl's theorem if the complement in the spectrum of the B-Weyl spectrum is the set of all eigenvalues which are isolated points of the spectrum. We prove that generalized Weyl's theorem holds for several classes of operators, extending previous results of Istrătescu and Curto-Han. We also consider the preservation of generalized Weyl's theorem between two operators $T \in L(X), S \in L(Y)$ intertwined or asymptotically intertwined by a quasi-affinity $A \in L(X, Y)$.
\end{abstract}

1. Introduction. If $T \in L(X)$, where $L(X)$ denotes the Banach algebra of all bounded linear operators on a complex Banach space $X$, we denote by $N(T)$ its kernel and by $R(T)$ its range. The operator $T \in L(X)$ is called a $B$-Fredholm operator [9] if there is an integer $n$ for which $R\left(T^{n}\right)$ is closed and the operator $T_{n}: R\left(T^{n}\right) \rightarrow R\left(T^{n}\right)$ defined by $T_{n}(x)=T(x)$ for every $x \in R\left(T^{n}\right)$ is a Fredholm operator. From [10, Theorem 3.1], it follows that $T$ is a B-Fredholm operator if and only if there exists an integer $n$ such that $c_{n}(T)<\infty$ and $c_{n}^{\prime}(T)<\infty$, where $c_{n}(T):=\operatorname{dim}\left(R\left(T^{n}\right) / R\left(T^{n+1}\right)\right)$ and $c_{n}^{\prime}(T):=\operatorname{dim}\left(N\left(T^{n+1}\right) / N\left(T^{n}\right)\right)$. For a B-Fredholm operator $T \in L(X)$ the index is defined by $\operatorname{ind}(T)=c_{n}^{\prime}(T)-c_{n}(T)$. From [9, Proposition 2.1], the index is independent of the choice of the integer $n$. Moreover, in the case of a Fredholm operator, this definition coincides with the classical definition of the index. Recall that the ascent of $T$ is defined as $a(T)=: \inf \{n \in \mathbb{N}$ : $\left.N\left(T^{n}\right)=N\left(T^{n+1}\right)\right\}$, while the descent is defined as $\delta(T):=\inf \{n \in \mathbb{N}$ : $\left.R\left(T^{n}\right)=R\left(T^{n+1}\right)\right\}$ (in both cases the infimum over the empty set is taken to be $\infty)$. An operator $T \in L(X)$ is said to be left Drazin invertible if $a(T)<\infty$ and $R\left(T^{a(T)+1}\right)$ is closed. Note that if $T$ has finite ascent and finite descent then $a(T)=\delta(T)$ (see [1, Theorem 3.3]). It is well known that $0<a(T-\lambda I)=\delta(T-\lambda I)<\infty$ precisely when $\lambda$ is a pole of the resolvent of $T$. A Fredholm operator which has index 0 is called a Weyl operator, and

2010 Mathematics Subject Classification: Primary 47A11, 47A53; Secondary 47A10. Key words and phrases: localized SVEP, B-Fredholm theory, Weyl's theorems, quasiaffinity, commutator. 
the Weyl spectrum is defined as $\sigma_{\mathrm{W}}(T)=\{\lambda \in \mathbb{C}: T-\lambda I$ is not Weyl $\}$. These concepts may be generalized ad follows:

Definition 1.1. Let $T \in L(X)$. Then $T$ is called a $B$-Weyl operator if it is a B-Fredholm operator of index 0 . The $B$-Weyl spectrum $\sigma_{\mathrm{BW}}(T)$ of $T$ is defined by $\sigma_{\mathrm{BW}}(T)=\{\lambda \in \mathbb{C}: T-\lambda I$ is not a $\mathrm{B}$-Weyl operator $\}$.

A classical result of Weyl on the fine structure of the spectrum of a normal operator $T$, defined on a Hilbert space, states that $\sigma_{\mathrm{W}}(T)=\sigma(T) \backslash E_{0}(T)$, where $E_{0}(T)$ denotes the set of isolated eigenvalues of finite multiplicity. This result has been extended to many other important classes of operators, and in the literature $T$ is said to satisfy Weyl's theorem if $\sigma_{\mathrm{W}}(T)=$ $\sigma(T) \backslash E_{0}(T)$. By [10. Theorem 4.5] any normal operator $T$ also satisfies $\sigma_{\mathrm{BW}}(T)=\sigma(T) \backslash E(T)$, where $E(T)$ is the set of all eigenvalues of $T$ which are isolated points of $\sigma(T)$, and in general $T \in L(X)$ is said to satisfy generalized Weyl's theorem if $\sigma_{\mathrm{BW}}(T)=\sigma(T) \backslash E(T)$. Note that

$$
\text { generalized Weyl's theorem } \Rightarrow \text { Weyl's theorem, }
$$

and the opposite implication is, in general, not true (see [13, Theorem 3.9]).

Two weaker variants of Weyl's theorems are defined as follows: $T \in L(X)$ satisfies Browder's theorem if $\sigma_{\mathrm{W}}(T)=\sigma(T) \backslash \Pi_{0}(T)$, where $\Pi_{0}(T)$ is the set of those poles $\lambda \in \mathbb{C}$ of the resolvent of $T$ such that $T-\lambda$ is Fredholm (or equivalently Weyl, see [1, Theorem 3.4]). A bounded operator $T \in L(X)$ is said to satisfy generalized Browder's theorem if $\sigma_{\mathrm{BW}}(T)=\sigma(T) \backslash \Pi(T)$, where $\Pi(T)$ denotes the set of all poles of the resolvent of $T$. Note that, in general, $\Pi(T) \subseteq E(T)$ for every $T \in L(X)$. Recently, it has been proved that Browder's theorem and generalized Browder's theorem for an operator are equivalent ([8]). Browder's theorem and Weyl's theorem are related as follows (see [11, Corollary 2.6] and [2, Theorem 3.1]):

Theorem 1.2. Let $T \in L(X)$. Then $T$ satisfies Weyl's theorem if and only if Browder's theorem holds for $T$ and $E_{0}(T)=\Pi_{0}(T)$. Analogously, $T$ satisfies generalized Weyl's theorem if and only if generalized Browder's theorem (or, equivalently, Browder's theorem) holds for $T$ and $E(T)=\Pi(T)$.

In this paper we prove that generalized Weyl's theorem holds for several classes of operators, extending earlier results of [24] and [15]. In particular, we give sufficient and necessary conditions for a finitely ascensive operator to obey generalized Weyl's theorem. The preservation of Weyl's theorem when $T \in L(X)$ and $S \in L(Y)$ are intertwined by a quasi-affinity has been investigated in 25. Part of this paper concerns the preservation of generalized Weyl's theorem for operators intertwined by a quasi-affinity. We shall also consider the more general case where $T$ and $S$ are asymptotically intertwined by a quasi-affinity. 
2. Generalized Weyl's theorem. The following lemma will be needed.

Theorem 2.1. If $T \in L(X)$ and $a(T)<\infty$ then the following statements are equivalent:

(i) There exists $n \geq a(T)+1$ such that $T^{n}(X)$ is closed;

(ii) There exists $p$ such that $T^{n}(X)$ is closed for all $n \geq p$.

Proof. We have $k_{i}(T):=c_{i}^{\prime}(T)-c_{i+1}^{\prime}(T)=0$ for all $i \geq a(T)$. The equivalence then easily follows from [29, Lemma 12].

Definition 2.2. We say that $T \in L(X)$ has the single valued extension property at $\lambda_{0} \in \mathbb{C}$ if for every open neighborhood $U$ of $\lambda_{0}, f=0$ is the only analytic function $f: U \rightarrow X$ such that $(T-\lambda I) f(\lambda)=0$ for all $\lambda \in U$. We will say that $T$ has the single valued extension property (SVEP) if $T$ has this property at every $\lambda \in \mathbb{C}$.

Note that

$$
a(T-\lambda I)<\infty \Rightarrow T \text { has SVEP at } \lambda,
$$

and the converse holds if $T$ is B-Fredholm (see [3]).

Of importance in local spectral theory is the glocal spectral subspace $\mathcal{X}_{T}(F)$ associated with a closed subset $F \subseteq \mathbb{C}$. It is defined, for all $T \in L(X)$, as the set of all $x \in X$ for which there exists an analytic function $f: \mathbb{C} \backslash F$ $\rightarrow X$ which satisfies the identity

$$
(\lambda I-T) f(\lambda)=x \quad \text { for all } \lambda \in \mathbb{C} \backslash F .
$$

Recall that a bounded operator $T \in L(X), X$ a Banach space, is said to have Dunford's property $(C)$, briefly property $(C)$, if, for each closed set $F \subseteq \mathbb{C}$, $\mathcal{X}_{T}(F)$ is closed. It is well-known that property $(C)$ implies SVEP.

If $T \in L(X)$, the quasi-nilpotent part of $T$ is defined by

$$
H_{0}(T):=\left\{x \in X:\left\|T x^{n}\right\|^{1 / n} \rightarrow 0\right\} .
$$

Note that $N\left(T^{n}\right) \subseteq H_{0}(T)$ for all $n \in \mathbb{N}$. Moreover, $H_{0}(T-\lambda I)=\mathcal{X}_{T}(\{\lambda\})$ (see [1, Theorem 2.20]). The analytical core of $T$ is defined by $K(T):=$ $\left\{x \in X\right.$ : there exist $c>0$ and a sequence $\left(x_{n}\right)_{n \geq 1} \subseteq X$ such that $T x_{1}=x$, $T x_{n+1}=x_{n}$ for all $n \in \mathbb{N}$, and $\left\|x_{n}\right\| \leq c^{n}\|x\|$ for all $\left.n \in \mathbb{N}\right\}$. Note that $T(K(T))=K(T)$.

If $\lambda$ is isolated in $\sigma(T)$, then it is known that $H_{0}(T-\lambda I)$ and $K(T-\lambda I)$ are closed subspaces of $X$, since $H_{0}(T-\lambda I)$ coincides with the range of the spectral projection $P_{\lambda}$ associated with $\{\lambda\}$, and $K(T-\lambda I)$ coincides with the kernel of $P_{\lambda}$ ([1, Theorem 3.74]). Therefore, $X=H_{0}(T-\lambda I) \oplus K(T-\lambda I)$, where $\oplus$ means topological direct sum. If $\lambda$ is a pole of the resolvent and $p:=a(T-\lambda I)=\delta(T-\lambda I)<\infty$ then $H_{0}(T-\lambda I)=N\left((T-\lambda I)^{p}\right)$ and $K(T-\lambda I)=R\left((T-\lambda I)^{p}\right)$. 
An operator $T$ in $B(X)$ is totally paranormal if

$$
\|(T-\lambda I) x\|^{2} \leq\left\|(T-\lambda I)^{2} x\right\|\|x\| \quad \text { for all } x \in X, \lambda \in \mathbb{C} .
$$

TheOREM 2.3. Let $T$ in $B(X)$ be a totally paranormal operator. Then generalized Weyl's theorem holds for $T$.

Proof. From [26] we know that if $T$ is totally paranormal, then $T-\lambda I$ has finite ascent for all $\lambda \in \mathbb{C}$. Hence $T$ has SVEP. Also from [26, Corollary 4.8], we have $H_{0}(T-\lambda I)=N(T-\lambda I)$ for all $\lambda \in \mathbb{C}$. From [12, Theorem 3.5], it then follows that $T$ satisfies generalized Weyl's theorem.

A spectral point $\lambda \in \sigma(T)$ is said to be a bare point if it lies on the circumference of some closed disc that contains $\sigma(T)$.

Theorem 2.4. Suppose that $T \in L(X)$ and every $\lambda \in \sigma(T)$ is a bare point. Then T has SVEP.

Proof. Let $U \subseteq \mathbb{C}$ be an nonempty open set and let $f: U \rightarrow X$ be an analytic function such that $(T-\lambda I) f(\lambda)=0$ for all $\lambda \in U$. Let $\rho(T):=$ $\mathbb{C} \backslash \sigma(T)$ be the resolvent set of $T$. Since every spectral point of $T$ is bare, $U \cap \rho(T) \neq \emptyset$. Therefore there exists an open nonempty subset $V$ contained in $U \cap \rho(T)$. Moreover for all $\lambda \in V$, we have $f(\lambda)=0$, because $T-\lambda I$ is invertible. Since the zeros of a nonvanishing analytic function are isolated, $f=0$. Hence $T$ has SVEP.

Let $T \in L(X)$ and $m \in \mathbb{N}$. We say that $T$ satisfies the growth condition $\left(G_{m}\right)$ if

$$
\sup _{\lambda \notin \sigma(T)}\left\|(T-\lambda I)^{-1}\right\| \operatorname{dist}(\lambda, \sigma(T))^{m}<\infty .
$$

An operator $T \in L(X)$ is said to be isoloid if every isolated point of the spectrum is an eigenvalue. In [16, Lemma 3] it is shown that every $T \in L(X)$ which satisfies the growth condition $\left(G_{m}\right)$ for some $m$ is polaroid (i.e. every isolated point of the spectrum is a pole) and hence isoloid. Let $\mathcal{H}(\sigma(T))$ denote the set of all analytic functions defined on an open neighborhood of $\sigma(T)$ and define, by the classical functional calculus, $f(T)$ for every $f \in$ $\mathcal{H}(\sigma(T))$.

Corollary 2.5. Suppose that $T \in L(X)$ has SVEP and satisfies the growth condition $\left(G_{m}\right)$ for some $m$. Then $f(T)$ satisfies generalized Weyl's theorem for each $f \in \mathcal{H}(\sigma(T))$. In particular, if $T$ satisfies $\left(G_{m}\right)$ and every $\lambda \in \sigma(T)$ is a bare point then generalized Weyl's theorem holds for $f(T)$ for each $f \in \mathcal{H}(\sigma(T))$.

Proof. Generalized Weyl's theorem for $T$ is a direct consequence of [12, Corollary]. Since $T$ satisfies SVEP the spectral theorem holds for $\sigma_{\mathrm{BW}}(T)$. Moreover, as $T$ is isoloid, by [15, Theorem 3.4] generalized Weyl's theorem 
holds for $f(T)$ for each $f \in \mathcal{H}(\sigma(T))$. The last statement is clear from Theorem 2.4.

As noted above, an operator satisfying generalized Weyl's theorem also satisfies Weyl's theorem. Corollary 2.5 extends an earlier result of Istrățescu 24. Theorem], where Weyl's theorem was proved for bounded operators $T$ for which condition $\left(G_{1}\right)$ holds for the restriction $(T-\lambda I) \mid M$ to every closed invariant subspace $M$ and such that the bare point condition is satisfied. Observe that if the Banach space $X$ is reflexive (in particular, a Hilbert space), the growth condition $\left(G_{m}\right)$ for $T \in L(X)$ entails property $(C)$, and consequently that $T$ has SVEP ([28, Proposition 1.2.19]). Since $T$ is polaroid, we have $E(T)=\Pi(T)$, hence $T$ satisfies generalized Weyl's theorem. The argument of the proof of Corollary 2.5 then also works, so $f(T)$ satisfies generalized Weyl's theorem, and in the case of reflexive Banach spaces, the bare point condition is not necessary.

Corollary 2.6. Let $T \in L(X)$ be a spectral operator of finite type. Then $f(T)$ satisfies generalized Weyl's theorem for each $f \in \mathcal{H}(\sigma(T))$.

Proof. If $T \in L(H)$ is a spectral operator of finite type, then from 18, Theorem XV 6.7], $T$ satisfies the growth condition $\left(G_{m}\right)$ for some $m$. Moreover, $T$ has SVEP.

Corollary 2.6 extends [30, Theorem 4] where it is proved that Weyl's theorem holds for every spectral operator of finite type.

An operator $T \in L(X)$ is said to be reguloid (see [23]) if for each isolated point $\lambda \in \sigma(T)$ the operator $T-\lambda I$ is regular in the sense that there exists an operator $S \in B(X)$ such that $T-\lambda I=(T-\lambda I) S(T-\lambda I)$.

THEOREM 2.7. If $T \in B(X)$ has SVEP and is reguloid, then generalized Weyl's theorem holds for $T$ if and only if for all $\lambda \in E(T)$ the sequence $\left(N(T-\lambda I) \cap R\left((T-\lambda I)^{n}\right)\right)_{n}$ is stationary for $n$ large enough.

Proof. If $T$ satisfies generalized Weyl's theorem, and $\lambda \in E(T)$, then $\lambda \in \Pi(T)$. So $T-\lambda I$ has a finite ascent and descent. Consequently, the sequence $\left(N(T-\lambda I) \cap R\left((T-\lambda I)^{n}\right)\right)_{n}$ is stationary for $n$ large enough. Conversely, let $\lambda \in E(T)$. Then $\lambda$ is an isolated point in $\sigma(T)$ and by our assumption $T-\lambda$ is a regular operator. In this case it is well known ([22, Theorem 3.8.2]) that $R\left((T-\lambda I)^{n}\right)$ is closed for each integer $n$. As the sequence $\left(N(T-\lambda I) \cap R\left((T-\lambda I)^{n}\right)\right)_{n}$ is stationary for $n$ large enough, $T-\lambda I$ is of topological uniform descent ([21]). Since $T$ has SVEP, from [12, Theorem 3.5] it follows that $T$ satisfies generalized Weyl's theorem.

It should be noted that $\left(N(T-\lambda I) \cap R\left((T-\lambda I)^{n}\right)\right)$ is a stationary sequence for $n$ large enough in the following cases: 
(a) $\operatorname{dim} N(T-\lambda I)<\infty$ or $\operatorname{cod} R(T-\lambda I)<\infty$ (see [1, proof of Theorem $1.10])$

(b) $a(T-\lambda I)<\infty$ or $\delta(T-\lambda I)<\infty$ (see [1, proof of Theorem 3.5]).

An operator $T \in L(X)$ is said to be finitely ascensive if $a(T-\lambda I)<\infty$ for all $\lambda \in \mathbb{C}$.

TheOREM 2.8. Let $T \in L(X)$ be a finitely ascensive operator. Then the following statements are equivalent:

(i) T satisfies generalized Weyl's theorem.

(ii) $E(T)=\Pi(T)$.

(iii) For every $\lambda \in E(T)$ there exists an integer $p:=p(\lambda)$ such that $R\left((T-\lambda I)^{n}\right)$ is closed for all $n \geq p$.

(iv) For every $\lambda \in E(T)$ there exists an integer $p:=p(\lambda)$ such that $H_{0}(T-\lambda I)=N\left((T-\lambda I)^{p}\right)$.

Proof. (i) $\Leftrightarrow$ (ii) is clear by Theorem 1.2 , since every finitely ascensive operator has SVEP and hence satisfies generalized Browder's theorem ([5, Corollary 3.3]).

(ii) $\Leftrightarrow($ iii). If $\lambda \in E(T)=\Pi(T)$ then $\lambda$ is an isolated spectral point and $p:=a(T-\lambda I)=\delta(T-\lambda I)<\infty$. If $n \geq p$ then $R\left((T-\lambda I)^{n}\right)=R\left((T-\lambda I)^{p}\right)$ is closed, since it coincides with the kernel of the spectral projection associated with $\{\lambda\}$. Conversely, assume that for all $\lambda \in E(T)$ there exists $p \in \mathbb{N}$ such that $R\left((T-\lambda I)^{n}\right)$ is closed for all $n \geq p$. Since $a(T-\lambda I)<\infty$, from Lemma 2.1 we know that $R\left((T-\lambda I)^{a(T)+1}\right)$ is closed, so $T-\lambda I$ is left Drazin invertible, and hence semi-B-Fredholm. Since $\lambda$ is isolated in $\sigma(T)=\sigma\left(T^{*}\right)$, $T^{*}$ has SVEP at $\lambda$ and hence (see [3, Theorem 2.11]) we have $\delta(T-\lambda I)<\infty$. Therefore, $\lambda$ is a pole of the resolvent and hence $\lambda \in \Pi(T)$. Consequently, $E(T)=\Pi(T)$.

(ii) $\Leftrightarrow($ iv). Suppose that $E(T)=\Pi(T)$. If $\lambda \in E(T)$ then $\lambda$ is a pole of the resolvent and hence $H_{0}(T-\lambda I)=N\left((T-\lambda I)^{p}\right)$, where $p:=a(T-\lambda I)=$ $\delta(T-\lambda I)$. Conversely, let $\lambda \in E(T)$ and suppose that we have $H_{0}(T-\lambda I)=$ $N\left((T-\lambda I-T)^{p}\right)$ for some $p \in \mathbb{N}$. Since $\lambda$ is an isolated point of $\sigma(T)$,

$$
X=H_{0}(T-\lambda) \oplus K(T-\lambda I)=N\left((T-\lambda I)^{p}\right) \oplus K(T-\lambda I),
$$

from which we obtain

$$
R\left((T-\lambda I)^{p}\right)=(T-\lambda I)(K(T-\lambda I))=K(T-\lambda I) .
$$

Thus $X=N\left((T-\lambda I)^{p}\right) \oplus R\left((T-\lambda I)^{p}\right)$ and this implies that $\lambda$ is a pole of the resolvent, i.e. $\lambda \in \Pi(T)$. Consequently, $E(T)=\Pi(T)$.

TheOREM 2.9. Suppose that $T \in L(X)$ is a finitely ascensive operator and isoloid. Then $f(T)$ satisfies generalized Weyl's theorem if and only if $T$ does. 
Proof. Suppose that $T$ satisfies generalized Weyl's theorem. Moreover, $T$ has SVEP and hence the spectral theorem holds for $\sigma_{\mathrm{BW}}(T)$ (see [5, Theorem 3.4]). By Theorem 3.4 of [15], $f(T)$ satisfies generalized Weyl's theorem for every $f \in \mathcal{H}(\sigma(T))$. The converse is clear.

The class of isoloid operators which are finitely ascensive is rather large. In [31] the class of $H(p)$-operators was defined to consist of all $T \in L(X)$ such that for all $\lambda \in \mathbb{C}$ there exists an integer $p:=p(\lambda)$ such that $H_{0}(T-\lambda I)=$ $N\left((T-\lambda I)^{p}\right)$. Property $H(p)$ is satisfied by every subscalar operator, and in particular for $p$-hyponormal, log-hyponormal, and $M$-hyponormal operators on Hilbert spaces ([31]). Furthermore, every multiplier of a commutative semisimple Banach algebra is $H(1)$ (see [1, Theorem 4.33]).

Evidently, every $H(p)$-operator is finitely ascensive, since $H_{0}(T-\lambda I$ $-T)=N\left((T-\lambda I)^{p}\right)=N\left((T-\lambda I)^{n}\right)$ for every $n \geq p$, hence by Theorem 2.8 the generalized Weyl's theorem holds for $T$. Every $H(p)$-operator $T$ is polaroid and hence isoloid ([2]), so, by Theorem 2.9, $f(T)$ satisfies generalized Weyl's theorem for all $f \in \mathcal{H}(\sigma(T))$.

We can say much more. Recall that a bounded operator is said to be algebraically $H(p)$ if there exists a nonconstant polynomial $p$ such that $p(T)$ is $H(p)$. Analogously, $T \in L(X)$ is said to be algebraically paranormal if there exists a nonconstant polynomial $p$ such that $p(T)$ is paranormal.

THEOREM 2.10. If $T \in L(X)$ is an algebraically $H(p)$-operator then $f(T)$ satisfies generalized Weyl's theorem for all $f \in \mathcal{H}(\sigma(T))$. Analogously, if $L(H), H$ a Hilbert space, is algebraically paranormal then $f(T)$ satisfies generalized Weyl's theorem for all $f \in \mathcal{H}(\sigma(T))$.

Proof. Suppose that $h(T)$ is an $H(p)$-operator for some nonconstant polynomial $h$. Then $h(T)$ has SVEP and hence $T$ has SVEP ([1, Theorem 2.40]). Therefore, generalized Browder's theorem holds for $T$ ([5]). Furthermore, $T$ is polaroid (see the proof of [17, Lemma 3.6]), so that $E(T)=\Pi(T)$. By Theorem 1.2 every $H(p)$-operator satisfies generalized Weyl's theorem. On the other hand, $T$ is isoloid and since the spectral theorem holds for $\sigma_{\mathrm{BW}}(T)$, by [15, Theorem 3.4] generalized Weyl's theorem holds for $f(T)$ for each $f \in \mathcal{H}(\sigma(T))$.

The proof in the case where $T \in L(H)$ is algebraically paranormal is analogous: every algebraically paranormal operator on a Hilbert space has $\operatorname{SVEP}([6])$, and is polaroid by [2, Lemma 4.3].

Every $M$-hyponormal operator is an $H(p)$-operator, so Theorem 2.10 subsumes the result of [15, Theorem 4.7] and extends [17, Theorem 3.1]. Generalized Weyl's theorem for $f(T)$ has been proved in [15, Theorem 4.14] by using different arguments. 
A closed subspace $M$ of $X$ reduces an operator $T \in L(X)$ if there is a closed subspace $N$ of $X$ such that $X=M \oplus N$ and both $M$ and $N$ are invariant under $T$.

TheOREM 2.11. Suppose that $T \in L(X)$ is reduced by the eigenspaces $N\left((T-\lambda I)^{k}\right)$ for all $k \geq p$ and all $\lambda \in \mathbb{C}$. Then $T$ satisfies generalized Weyl's theorem if and only if any one of the conditions (ii)-(iv) of Theorem 2.8 holds.

Proof. By Theorem 2.8 it suffices to prove that $T$ is finitely ascensive, i.e. $T-\lambda I$ is of finite ascent for all $\lambda \in \mathbb{C}$. By assumption for any $k \geq p$, and all $\lambda \in \mathbb{C}$, there is an invariant subspace $M$ of $T$ such that we have $X=N\left((T-\lambda I)^{k}\right) \oplus M$. Suppose $x \in N\left((T-\lambda I)^{k+1}\right)$ and write $x=x_{1}+x_{2}$, with $x_{1} \in N\left((T-\lambda I)^{k}\right)$ and $x_{2} \in M$. Then

$(T-\lambda I)^{k+1} x=(T-\lambda I)^{k+1} x_{1}+(T-\lambda I)^{k+1} x_{2}=(T-\lambda I)^{k}(T-\lambda I) x_{2}=0$.

So, $(T-\lambda I) x_{2} \in N\left((T-\lambda I)^{k}\right) \cap M$ and then $(T-\lambda I) x_{2}=0$. Hence $x_{2} \in N(T-\lambda I) \subseteq N\left((T-\lambda I)^{k}\right)$ and $x \in N\left((T-\lambda I)^{k}\right)$. It follows that $N\left((T-\lambda I)^{k+1}\right) \subseteq N\left((T-\lambda I)^{k}\right)$ for any $k \geq p$. The opposite inclusion holds for every operator, so $N\left((T-\lambda I)^{k+1}\right)=N\left((T-\lambda I)^{k}\right)$. Hence, $T$ is finitely ascensive.

Clearly, the assumption of Theorem 2.11 is satisfied if $T$ is reduced by each of eigenspaces.

3. Generalized Weyl's theorem and quasi-affinity. In this section we study the problem of preserving generalized Weyl's theorem from a bounded operator $S \in L(Y)$ to $T \in L(X)$ when $T$ and $S$ are intertwined by a quasi-affinity.

Lemma 3.1. Suppose that $S \in L(Y)$ has SVEP at $\lambda_{0}$, and let $T \in L(X)$ be such that there exists $A \in L(X, Y)$ injective for which $S A=A T$. Then $T$ has SVEP at $\lambda_{0}$.

Proof. Let $U \subseteq \mathbb{C}$ be an open neighborhood of $\lambda_{0}$ and $f: U \rightarrow X$ be an analytic function such that $(T-\lambda I) f(\lambda)=0$ for all $\lambda \in U$. Then

$$
(S-\lambda I) A f(\lambda)=A(T-\lambda I) f(\lambda)=0 \quad \text { for all } \lambda \in U .
$$

Since $S$ has SVEP at $\lambda_{0}$ we have $A f(\lambda)=0$, and since $A$ is injective we conclude that $f(\lambda)=0$ for all $\lambda \in U$. Therefore $T$ has SVEP at $\lambda_{0}$.

Definition 3.2. The operator $A \in L(X, Y)$ between the Banach spaces $X$ and $Y$ is a quasi-affinity if it has a trivial kernel and dense range. We say that $T \in L(X)$ is a quasi-affine transform of $S \in L(Y)$, and we write $T \prec S$, if there is a quasi-affinity $A \in L(X, Y)$ that intertwines $T$ and $S$, i.e. $S A=A T$. If there exist two quasi-affinities $A \in L(X, Y), B \in L(X, Y)$ for which $S A=A T$ and $B S=T B$ then we say that $S$ and $T$ are quasi-similar. 
Theorem 3.3. Let $T \in L(X)$ and $S \in L(Y)$ be such that $S$ has $S V E P$. Then the following statements hold:

(i) If $T$ and $S$ are intertwined by an injective map $A \in L(X, Y)$ and $E(T) \subseteq E(S)$ then generalized Weyl's theorem for $S$ implies generalized Weyl's theorem for $T$.

(ii) If $T \prec S, \sigma(S) \subseteq \sigma(T)$, and $S$ satisfies generalized Weyl's theorem then so does $T$.

Proof. (i) If $S$ has SVEP then $T$ has SVEP, by Lemma 3.1, so generalized Browder's theorem holds for $T$. By Theorem 1.2 it suffices to prove $E(T)=$ $\Pi(T)$. Let $\lambda \in E(T)$. Then $\lambda \in E(S)$ and since $S$ satisfies generalized Weyl's theorem, $\lambda \in \Pi(S)$. Let $P_{S}(\lambda)$ and $P_{T}(\lambda)$ denote, respectively, the spectral projections associated with the spectral set $\{\lambda\}$ for $S$ and $T$. From [19, Lemma 2.1], we have $P_{S}(\lambda) A=A P_{T}(\lambda)$. Since $\lambda \in \Pi(S)$, we have $p:=a(S-\lambda I)=\delta(S-\lambda I)<\infty$ and $N\left((S-\lambda I)^{p}\right)=R\left(P_{S}(\lambda)\right)$ (see [1, Theorem 3.74]). Therefore, $(S-\lambda I)^{p} P_{S}(\lambda)=0$, and consequently

$$
0=(S-\lambda I)^{p} P_{S}(\lambda) A=A(T-\lambda I)^{p} P_{T}(\lambda) .
$$

Since $A$ is injective, we have $(T-\lambda I)^{p} P_{T}(\lambda)=0$, and hence $R\left(P_{T}(\lambda)\right) \subseteq$ $N\left((T-\lambda I)^{p}\right)$. The opposite inclusion is obvious, since the range of $P_{T}(\lambda)$ is the quasi-nilpotent part of $T-\lambda I$. Hence $R\left(P_{T}(\lambda)\right)=N\left((T-\lambda I)^{p}\right)$. Arguing as in the last part of the proof of Theorem 2.8 we deduce that $Y=N\left((T-\lambda I)^{p}\right) \oplus R\left((T-\lambda I)^{p}\right)$, from which it follows that $\lambda \in \Pi(T)$. Therefore $E(T)=\Pi(T)$.

(ii) The statement follows from (i) once we prove that $\sigma(S) \subseteq \sigma(T)$ entails $E(T) \subseteq E(S)$. Let $\lambda \in E(T)$. Since $T \prec S$, from [19, Theorem 2.5] it follows that $\lambda \in \sigma(S)$. As $\sigma(S) \subseteq \sigma(T), \lambda$ is isolated in $\sigma(S)$. It is easily seen that $\lambda$ is an eigenvalue of $S$. Indeed, let $A \in L(X, Y)$ be a quasi-affinity for which $S A=A T$. Since $\lambda$ is an eigenvalue of $T$ there exists an $x \neq 0$ such that $(T-\lambda I) x=0$. Then

$$
(S-\lambda I) A x=A(T-\lambda I) x=0,
$$

and since $A$ is injective we have $A x \neq 0$, so $A x$ is an eigenvector of $S-\lambda I$. Therefore, $\lambda \in E(S)$ and hence $E(T) \subseteq E(S)$, as desired.

It is not surprising that, if $T \prec S$, the preservation of generalized Weyl's theorem requires some spectral inclusions to be satisfied. If $T \prec S$ a classical result due to Rosenblum shows that $\sigma(S)$ and $\sigma(T)$ must overlap (see [20]). But also the stronger condition of quasi-similarity is, in general, not sufficient to preserve the spectrum. This happens only in some special cases, for instance if $T$ and $S$ are quasi-similar hyponormal operators, or whenever $T$ and $S$ have totally disconnected spectra (see [20, Corollary 2.5]). 
We now introduce some important notions from local spectral theory. Let $U$ be an open subset of $\mathbb{C}$ and denote by $\mathcal{H}(U, X)$ the Fréchet space of all analytic functions $f: U \rightarrow X$ with respect the pointwise vector space operations and the topology of locally uniform convergence. $T \in L(X)$ has Bishop's property $(\beta)$ if, for every open $U \subseteq \mathbb{C}$ and every sequence $\left(f_{n}\right) \subseteq \mathcal{H}(U, X)$ for which $(\lambda I-T) f_{n}(\lambda)$ converges to 0 uniformly on every compact subset of $U$, also $f_{n} \rightarrow 0$ in $\mathcal{H}(U, X)$. An important class of operators with property $(\beta)$ is the class of subnormal operators (i.e. restrictions of normal operators to closed invariant subspaces). Note that property $(\beta)$ implies property $(C)$ and hence SVEP (see [28, Proposition 1.2.19]).

A bounded operator $T \in L(X)$ has the decomposition property $(\delta)$ if $X=$ $\mathcal{X}_{T}(\bar{U})+\mathcal{X}_{T}(\bar{V})$ for every open cover $\{U, V\}$ of $\mathbb{C}$. Decomposable operators may be defined in several ways, for instance as satisfying property $(\beta)$ and property $(\delta)$ (see [28, Theorem 2.5.19] for relevant definitions). Note that property $(\delta)$ implies SVEP for $T^{\star}$. In fact $T$ has property $(\delta)$ if and only if $T^{*}$ has property $(\beta)$ (see [28, Theorem 2.5.19]).

Theorem 3.3 applies to operators $T$ and $S$ having a totally disconnected spectrum, since they are decomposable. This result may be generalized as follows:

Corollary 3.4. Suppose that $T \in L(X)$ and $S \in L(Y)$ are decomposable and $T \prec S$. If generalized Weyl's theorem holds for $S$ then it also holds for $T$.

Proof. By Theorem 2.4.4 of [14] if $T$ and $S$ are decomposable and $T \prec S$ then $\sigma(T)=\sigma(S)$. Moreover, $S$ has SVEP.

Under the stronger condition of quasi-similarity the assumption of decomposability of Corollary 3.4 may be relaxed as follows:

Corollary 3.5. Let $T \in L(X)$ and $S \in L(Y)$ be quasi-similar.

(i) If $S$ has property ( $\beta)$ then generalized Weyl's theorem for $S$ implies generalized Weyl's theorem for $T$.

(ii) If $S$ is a Hilbert space operator for which property $(C)$ holds, then generalized Weyl's theorem for $S$ implies generalized Weyl's theorem for $T$.

Proof. (i) Suppose that $S$ satisfies generalized Weyl's theorem. If $S$ has property $(\beta)$ and $T, S$ are quasi-similar then, by a result of Putinar [32, $\sigma(T)=\sigma(S)$. Moreover, property $(\beta)$ entails SVEP and hence, by Theorem 3.3 , also $T$ satisfies generalized Weyl's theorem.

(ii) Property $(C)$ entails SVEP and also in this case we have $\sigma(T)=\sigma(S)$ (see [33]), so Theorem 3.3 applies.

The preservation of Weyl's theorem presents a simpler situation: 
TheOREM 3.6. Suppose that $T \in L(X)$ and $S \in L(Y)$ are intertwined by an injective map and $S$ has $S V E P$. If $E_{0}(T) \subseteq E_{0}(S)$ and $S$ satisfies Weyl's theorem, then so does $T$.

Proof. Since $T$ has SVEP we know that Browder's theorem holds for $T$. In order to show Weyl's theorem for $T$ it is enough, by Theorem 1.2, to prove $E_{0}(T)=\Pi_{0}(T)$, or equivalently, by [1, Theorem 3.84], that $H_{0}(T-\lambda I)$ is finite-dimensional for all $\lambda \in E_{0}(T)$.

Let $\lambda \in E_{0}(T)$. Then $\lambda \in E_{0}(S)$ and since $S$ satisfies Weyl's theorem, $E_{0}(S)=\Pi_{0}(S)$. Let $A \in L(X, Y)$ be an injective map such that $S A=A T$. Let $x \in H_{0}(T-\lambda I)$. Then

$\lim _{n \rightarrow \infty}\left\|(S-\lambda I)^{n} A x\right\|^{1 / n}=\lim _{n \rightarrow \infty}\left\|A(T-\lambda I)^{n} x\right\|^{1 / n} \leq \lim _{n \rightarrow \infty}\left\|(T-\lambda I)^{n} x\right\|^{1 / n}=0$, thus $A x \in H_{0}(S-\lambda I)$ and hence $A\left(H_{0}(T-\lambda I)\right) \subseteq H_{0}(S-\lambda I)$. We know $E_{0}(S)=\Pi_{0}(S)$ and it has been observed before that this equality is equivalent to $H_{0}(S-\lambda I)$ being finite-dimensional. Since $A$ is injective $H_{0}(T-\lambda I)$ is also finite-dimensional, so the proof is complete.

Since a finite ascensive operator has SVEP, the result of Theorem 3.6 shows that the hypothesis that $S$ is reduced by its eigenspaces in [25, Theorem 7] is not necessary.

We want now to study the preservation of generalized Weyl's theorem in the case that $T$ and $S$ are related by a very weak notion of intertwining which dates back to Foiaş (cf. [14, Chapter 4] and [28, Chapter 3]). If $T \in L(X)$ and $S \in L(Y)$, the commutator $C(S, T)$ is the mapping on $L(X, Y)$ defined by $C(S, T)(A):=S A-A T$ for all $A \in L(X, Y)$. An operator $A \in L(X, Y)$ is said to intertwine $T$ and $S$ asymptotically if

$$
\lim _{n \rightarrow \infty}\left\|C(S, T)^{n}(A)\right\|^{1 / n}=0,
$$

where by induction it is easy to show the binomial identity

$$
C(S, T)^{n}(A)=\sum_{k=0}^{n}\left(\begin{array}{l}
n \\
k
\end{array}\right)(-1)^{k} S^{n-k} A T^{k} .
$$

Evidently, this notion is a generalization of the intertwining condition $C(S, T)(A)=0$ which appears in the definition of $T \prec S$. This notion is also a generalization of the higher order intertwining condition:

$$
C(S, T)^{n}(A)=0 \quad \text { for some } n \in \mathbb{N} .
$$

Note that if $T$ and $S$ are generalized scalar then the condition (3.1) holds if and only if $C(S, T)^{n}(A)=0$ for some $n \in \mathbb{N}$ (see [14, Theorem 4.4.5]). If the pairs $(S, T)$ and $(T, S)$ are both asymptotically intertwined by some quasi-affinity then $T$ and $S$ are said to be asymptotically quasi-similar. We 
recall that if a pair $(S, T)$ is asymptotically intertwined by $A \in L(X, Y)$ then

$$
A\left(\mathcal{X}_{T}(F)\right) \subseteq \mathcal{Y}_{S}(F) \text { for all closed sets } F \subseteq \mathbb{C}
$$

(see Corollary 3.4.5 of [28]).

The result of Theorem 3.6 may be generalized as follows:

Theorem 3.7. Let $T \in L(X)$ and $S \in L(Y)$ be such that the pair $(S, T)$ is asymptotically intertwined by an injective map $A \in L(X, Y)$. Suppose that $T$ has $S V E P$ and $E_{0}(T) \subseteq E_{0}(S)$. If $S$ satisfies Weyl's theorem then so does $T$.

Proof. By Theorem 1.2 it suffices to prove $E_{0}(T)=\Pi_{0}(T)$, or equivalently that $H_{0}(T-\lambda I)$ is finite-dimensional for all $\lambda \in E_{0}(T)$. If $\lambda \in E_{0}(T)$ then $\lambda \in E_{0}(S)$ and since $S$ satisfies Weyl's theorem, $H_{0}(S-\lambda I)$ is finitedimensional. By $(3.2)$ we have

$$
A\left(H_{0}(T-\lambda I)\right)=A\left(\mathcal{X}_{T}(\{\lambda\})\right) \subseteq \mathcal{Y}_{S}(\{\lambda\})=H_{0}(S-\lambda I),
$$

and since $A$ is injective, $H_{0}(T-\lambda I)$ is finite-dimensional. Therefore, $T$ satisfies Weyl's theorem.

The analogue of Theorem 3.7 for generalized Weyl's theorem does not hold. To see that, consider a very particular case of asymptotically quasisimilar operators: $T, S \in L(X)$ are said to be quasi-nilpotent equivalent if each of the pairs $(S, T)$ and $(T, S)$ is asymptotically intertwined by the identity operator $I$ on $X$. Note that any quasi-nilpotent operator and the zero operator are quasi-nilpotent equivalent.

EXAmple 3.8. Generalized Weyl's theorem may fail for a quasi-nilpotent operator. For instance, if $T \in L\left(\ell^{2}(\mathbb{N})\right)$ is defined by

$$
T\left(x_{1}, x_{2}, \ldots\right)=\left(x_{2} / 2, x_{3} / 3, \ldots\right) \text { for all }\left(x_{n}\right) \in \ell^{2}(\mathbb{N}),
$$

then $T$ is quasi-nilpotent, so has SVEP, and

$$
\{0\}=E(T) \neq \sigma(T) \backslash \sigma_{\mathrm{BW}}(T)=\emptyset .
$$

Obviously, generalized Weyl's theorem holds for $S:=0$. Clearly, $E(T)=$ $E(0)=\{0\}$. This example shows that, in general, generalized Weyl's theorem is not transmitted whenever $S$ and $T$ are asymptotically intertwined by a quasi-affinity, even in the case that the inclusion $E(T) \subseteq E(S)$ is satisfied.

Theorem 3.9. Let $T \in L(X)$ and $S \in L(Y)$ be asymptotically intertwined by a quasi-affinity $A \in L(X, Y)$. Assume that $S$ is isoloid and $\Pi(S) \subseteq \Pi(T)$.

(i) If $S$ has property $(C)$, and if either $T$ or $T^{*}$ has $S V E P$, then generalized Weyl's theorem for $S$ implies that $T$ satisfies generalized Weyl's theorem. 
(ii) If $T$ and $S$ are asymptotically quasi-similar and each has one of the properties $(\delta)$ or $(C)$, then generalized Weyl's theorem for $S$ implies generalized Weyl's theorem for $T$.

Proof. (i) If $T$ or $T^{*}$ has SVEP then Browder's theorem (or equivalently, generalized Browder's theorem) holds for $T$ (see [5. Corollary 3.3]). By Theorem 1.2 it then suffices to prove $E(T)=\Pi(T)$. Let $\lambda \in E(T)$. Then $\lambda$ is an eigenvalue of $T$ and an isolated point of $\sigma(T)$. Since $A$ is injective, Corollary 3.5.8 of [28] entails that $\lambda \in \sigma(S)$ (more precisely, $\lambda$ belongs to the approximate-point spectrum of $S$ ). Since $A$ has dense range, property $(C)$ for $S$ entails, by [27, Theorem 4.1], that $\sigma(S) \subseteq \sigma(T)$. This implies that $\lambda$ is an isolated point of $\sigma(S)$, hence, by the isoloid condition, $\lambda$ is an eigenvalue of $S$, i.e. $\lambda \in E(S)$. Since $S$ satisfies generalized Weyl's theorem, we have $E(S)=\Pi(S) \subseteq \Pi(T)$, so $\lambda \in \Pi(T)$.

(ii) Note that $T$ or $T^{*}$ has SVEP, so Browder's theorem holds for $T$. By [28. Corollary 3.5.16] we know that $\sigma(T)=\sigma(S)$. If $\lambda \in E(T)$ then $\lambda$ is an isolated point of $\sigma(S)$, hence by the isoloid condition $\lambda \in E(S)=\Pi(S) \subseteq$ $\Pi(T)$.

REMARK 3.10. Without assuming the inclusion $\Pi(S) \subseteq \Pi(T)$, the results of Theorem 3.9 do not hold. For instance, the operators $S$ and $T$ of Example 3.8 do not satisfy this inclusion, since $\Pi(T)=\emptyset$ while $\Pi(S)=\{0\}$. Clearly, $S$ is isoloid, and also all the other assumptions on $T$ and $S$ are satisfied, since $T$ and $S$ are decomposable.

Note that if $T$ is polaroid then the assumption $\Pi(S) \subseteq \Pi(T)$ in part (ii) of Theorem 3.9 is redundant. In fact, from $\sigma(S)=\sigma(T)$ it follows that every $\lambda \in \Pi(S)$ is an isolated point of $\sigma(T)$, and hence a pole of $T$.

REMARK 3.11. It is easy to see that under the stronger assumption that $C(S, T)^{n}(A)=0$ for some $n \in \mathbb{N}$, the isoloid condition on $S$ in the statement of Theorem 3.9 is not required. To see this, observe first that every eigenvalue of $T$ is an eigenvalue of $S$. In fact, if $x \in X$ is an eigenvector for the eigenvalue $\lambda$ of $T$, then

$$
0=C(S, T)^{n}(A) x=C(S-\lambda I, T-\lambda I)^{n}(A) x=(S-\lambda I)^{n} A x,
$$

and since $A$ is injective we have $A x \neq 0$, so $(S-\lambda I)^{n}$ is not injective. This implies that $S-\lambda I$ is not injective either, so $\lambda$ is an eigenvalue of $S$. Now, we have seen in the proof of Theorem 3.9)(i) or (ii)) that if $\lambda \in E(T)$ then $\lambda$ is an isolated point of $\sigma(S)$. Moreover, $\lambda$ is an eigenvalue of $T$ and hence an eigenvalue of $S$, so $\lambda \in E(S)=\Pi(S) \subseteq \Pi(T)$.

Note that in Theorem 3.9 if we assume $S$ is decomposable, always under the assumption $C(S, T)^{n}(A)=0$, then the assumption that $T$ has SVEP can be dropped. In fact, $S$ has SVEP and it can be shown that SVEP carries over from $S$ to $T$. 
Corollary 3.12. Suppose that $T \in L(X)$ and $S \in L(Y)$ are decomposable operators which are asymptotically intertwined by a quasi-affinity. If $S$ is isoloid and $\Pi(S) \subseteq \Pi(T)$ then generalized Weyl's theorem for $S$ implies generalized Weyl's theorem for $T$.

Proof. $S$ has $(\delta)$, both $T$ and $T^{*}$ have SVEP. Hence Theorem 3.9 applies.

THeOREM 3.13. Suppose that $T, S \in L(X)$ are quasi-nilpotent equivalent, $S$ a polaroid operator which has $S V E P$, and $\Pi(S) \subseteq \Pi(T)$. Then generalized Weyl's theorem for $S$ implies generalized Weyl's theorem for $T$.

Proof. Observe first that quasi-nilpotent equivalence preserves SVEP and quasi-nilpotent equivalent operators have the same spectra (14, Theorems 2.2 and 2.3]). Moreover, by [27, Proposition 2.2] (taking $A=I$ ) we have $\mathcal{X}_{T}(F) \subseteq \mathcal{X}_{S}(F)$ for every closed subset $F$ of $\mathbb{C}$. Evidently, the opposite inclusion holds by symmetry. In particular, taking $F=\{\lambda\}$ we conclude that $H_{0}(T-\lambda I)=H_{0}(S-\lambda I)$ for every $\lambda \in \mathbb{C}$.

Now, let $\lambda \in E(T)$. Then $\lambda$ is an isolated point of $\sigma(T)=\sigma(S)$ and $T-\lambda I$ is not injective. Since $N(T-\lambda I) \subseteq H_{0}(T-\lambda I)$ it follows that $H_{0}(S-\lambda I)=H_{0}(T-\lambda I) \neq\{0\}$. Now, since $S$ is polaroid, there exists $p:=p(\lambda) \in \mathbb{N}$ such that $H_{0}(S-\lambda I)=N\left((S-\lambda I)^{p}\right)$ (see [4, Theorem 2.9]). If $S-\lambda I$ were injective then $(S-\lambda I)^{p}$ would also be injective and hence $H_{0}(S-\lambda I)=\{0\}$, which is impossible. Therefore, $\lambda \in E(S)$, i.e. $E(T) \subseteq$ $E(S)$, and by symmetry we conclude that $E(T)=E(S)$. Since $S$ satisfies generalized Weyl's theorem, we have $E(S)=\Pi(S)$ and hence $E(T) \subseteq \Pi(T)$, from which it follows that generalized Weyl's theorem holds for $T$.

The operators $T$ and $S$ of Example 3.8 show that without the assumption $\Pi(S) \subseteq \Pi(T)$, the result of Theorem 3.13 in general does not hold. A very particular case of quasi-nilpotent equivalence is when $C(S, T)^{n}(I)=0$ for some $n \in \mathbb{N}$. If $T$ and $S$ commute then $C(S, T)^{n}(I)=(S-T)^{n}=0$. In this case $T$ and $S$ differ by a commuting nilpotent operator $N$ and, without any condition, generalized Weyl's theorem is transmitted from $S$ to $T$ (see [7]).

Two operators $T \in L(X)$ and $S \in L(Y)$ are said to be asymptotically similar if there exists a bijection $A \in L(X, Y)$ such that $A$ intertwines $S$ and $T$ asymptotically and its inverse $A^{-1}$ intertwines $T$ and $S$ asymptotically. This is a slight generalization of quasi-nilpotent equivalence (see [14 for details).

TheOREM 3.14. Suppose that $T \in L(X)$ and $T \in L(Y)$ are asymptotically similar, $S$ a polaroid with $S V E P$, and $\Pi(S) \subseteq \Pi(T)$. Then generalized Weyl's theorem for $S$ implies generalized Weyl's theorem for $T$.

Proof. As noted before, asymptotic quasi-similarity, hence asymptotic similarity, preserves the spectrum and SVEP (see [27, Theorem 3.5]). There- 
fore, $T$ satisfies generalized Browder's theorem. To show $E(T)=\Pi(T)$ we proceed as in the proof of Theorem 3.13, taking into account the equality $A\left(H_{0}(T-\lambda I)\right)=H_{0}(S-\lambda I)$, always by [27, Proposition 2.2]. Therefore generalized Weyl's theorem for $S$ implies $E(T)=E(S)=\Pi(S) \subseteq \Pi(T)$.

Acknowledgments. Research of P.A. was supported by Fondi ex-60, 2007, Università di Palermo.

Research of M.B. was supported by Protars D11/16 and PGR-UMP.

\section{References}

[1] P. Aiena, Fredholm and Local Spectral Theory, with Application to Multipliers, Kluwer, 2004.

[2] - Classes of operators satisfying a-Weyl's theorem, Studia Math. 169 (2005), 105122.

[3] -, Quasi-Fredholm and localized SVEP, Acta Sci. Math. (Szeged) 73 (2007), 251263.

[4] P. Aiena, M. T. Biondi and F. Villafañe, Property $(w)$ and perturbations III, J. Math. Anal. Appl. 353 (2009), 205-214.

[5] P. Aiena and O. Garcia, Generalized Browder's theorem and SVEP, Mediterr. J. Math. 4 (2007), 215-228.

[6] P. Aiena and R. J. Guillen, Weyl's theorem for perturbations of paranormal operators, Proc. Amer. Math. Soc. 35 (2007), 2433-2442.

[7] M. Amouch and M. Berkani, Preservation of property (gw) under perturbations, Acta Sci. Math. (Szeged) 74 (2008), 764-781.

[8] M. Amouch and H. Zguitti, On the equivalence of Browder's and generalized Browder's theorem, Glasgow Math. J. 48 (2006), 179-185.

[9] M. Berkani, On a class of quasi-Fredholm operators, Integral Equations Operator Theory 34 (1999), 244-249.

[10] - Index of B-Fredholm operators and generalization of a Weyl theorem, Proc. Amer. Math. Soc. 130 (2002), 1717-1723.

[11] - B-Weyl spectrum and poles of the resolvent, J. Math. Anal. Appl. 272 (2002), 596-603.

[12] M. Berkani, N. Castro and S. V. Djordjević, Single valued extension property and generalized Weyl's theorem, Math. Bohem. 131 (2006), 29-38.

[13] M. Berkani and J. J. Koliha, Weyl type theorems for bounded linear operators, Acta Sci. Math. (Szeged) 69 (2003), 359-376.

[14] I. Colojoară and C. Foiaş, Theory of Generalized Spectral Operators, Gordon and Breach, New York, 1968.

[15] R. E. Curto and Y. M. Han, Generalized Browder's and Weyl's theorems for Banach space operators, J. Math. Anal. Appl. 336 (2007), 1424-1442.

[16] S. V. Djordjević, I. H. Jeon and E. Ko, Weyl's theorem through local spectral theory, Glasgow Math. J. 44 (2002), 323-327.

[17] B. P. Duggal, Weyl's theorem for algebraically totally hereditarily normaloid operators, J. Math. Anal. Appl. 308 (2005), 578-585.

[18] N. Dunford and J. T. Schwartz, Linear Operators, Part III: Spectral Operators, Wiley-Interscience, 1971. 
[19] L. A. Fialkow, A note on quasisimilarity of operators, Acta Sci. Math. (Szeged) 39 (1977), 67-85.

[20] S. Grabiner, Spectral consequences of the existence of intertwining operators, Comment. Math. Prace Mat. 22 (1980-81), 227-238.

[21] —, Uniform ascent and descent of bounded operators, J. Math. Soc. Japan 34 (1982), 317-337.

[22] R. Harte, Invertibility and Singularity for Bounded Linear Operators, Dekker, New York, 1988.

[23] R. Harte and W. Y. Lee, Another note on Weyl's theorem, Trans. Amer. Math. Soc. 349 (1997), 2115-2124.

[24] V. Istrăţescu, Weyl's theorem for a class of operators, Rev. Roumaine Math. Pures Appl. 13 (1968), 1103-1105.

[25] I. H. Jeon, Weyl's theorem and quasi-similarity, Integral Equations Operator Theory 39 (2001), 214-221.

[26] K. B. Laursen, Operators with finite ascent, Pacific J. Math. 157 (1992), 323-336.

[27] K. B. Laursen and M. M. Neumann, Asymptotic intertwining and spectral inclusions on Banach spaces, Czechoslovak Math. J. 43 (118) (1993), 483-497.

[28] —, Introduction to Local Spectral Theory, Clarendon Press, Oxford, 2000.

[29] M. Mbekhta and V. Müller, On the axiomatic theory of the spectrum, II, Studia Math. 119 (1996), 129-147.

[30] K. K. Oberai, On the Weyl's theorem, Illinois J. Math. 18 (1974), 208-212.

[31] M. Oudghiri, Weyl's and Browder's theorem for operators satisfying the SVEP, Studia Math. 163 (2004), 85-101.

[32] M. Putinar, Quasi-similarity of tuples with Bishop's property $(\beta)$, Integral Equations Operator Theory 15 (1992), 1047-1052.

[33] G. J. Stampfli, Quasi-similarity of operators, Proc. Roy. Irish Acad. 81 (1981), 109-119.

Pietro Aiena

Dipartimento di Metodi e Modelli Matematici

Facoltà di Ingegneria

Università di Palermo

Viale delle Scienze

I-90128 Palermo, Italy

E-mail: paiena@unipa.it
Mohammed Berkani Département de Mathématiques

Faculté des Sciences Université Mohammed I

Oujda, Morocco E-mail: berkani@sciences.univ-oujda.ac.ma 